\title{
Técnica y tratamiento en psicoanálisis. Una postura epistemológica
}

\section{Technique and treatment in psychoanalysis. An epistemological posture}

Julieta Bareiro*

\begin{abstract}
Resumen: Este trabajo parte de nuestra reflexión acerca de la tesis que Paul Ricoeur expone en su libro Freud. Una interpretación de la cultura, reflexión que se centra sólo en un aspecto del estatuto del psicoanálisis, a saber, en la caracterización que Ricoeur hace de la especificidad del mismo y su distinción radical de la fenomenología. A partir de allí, intentaremos mostrar que la recepción del psicoanálisis de Freud en Winnicott produjo un desplazamiento epistemológico cuando dejó de lado el concepto de técnica y lo reemplazó por la noción de tratamiento. Por ello, a nuestro juicio, desde el interior del pensamiento de Winnicott se torna necesario pensar nuevamente las relaciones epistemológicas entre fenomenología y psicoanálisis.
\end{abstract}

Palabras clave: Ricoeur, Winnicott, Freud

\begin{abstract}
This paper starts from our reflection on the thesis that Paul Ricoeur exposes in his book Freud and Philosophy. An Essay on Interpretation, a reflection that focuses only on one aspect of the status of psychoanalysis, namely, Ricoeur's characterization of its specificity and its radical distinction from phenomenology. From there, we will try to show that the reception of Freud's psychoanalysis in Winnicott produced an epistemological shift when he put aside the concept of technique and replaced it with the notion of treatment. Therefore, in our view, from within Winnicott's thinking it becomes necessary to think again about the epistemological relationships between phenomenology and psychoanalysis.
\end{abstract}

Keywords: Ricoeur, Winnicott, Freud

* Dra. en Psicología (Universidad de Buenos Aires, Argentina), Magister en Psicoanálisis (UBA, Argentina), Doctoranda en Filosofía (Universidad Nacional de Lanús, Argentina), Investigadora Adjunta del Consejo Nacional de Investigaciones Científicas y Técnicas (CONICET), Prof. Adjunta de "Psicología, Ética y DDHH" (Facultad de Psicología, UBA), Categoría II del Programa de Incentivos. Dirección electrónica: jumba75@,hotmail.com 


\section{Introducción}

El siguiente trabajo es una reflexión sobre una tesis que Paul Ricoeur expone en su libro Freud. Una interpretación de la cultura. Esa tesis corresponde al momento epistemológico de lectura de Freud. En tal sentido, Ricoeur declara ya en las primeras páginas que la intención fundamental de su interpretación del psicoanálisis se centra en el estatuto epistémico del discurso psicoanalítico: "Mi problema es el de la consistencia del discurso freudiano. Trátase, en primer lugar, de un problema epistemológico: ¿qué cosa es interpretar en psicoanálisis y cómo la interpretación de los signos humanos se articula con la explicación económica que pretende alcanzar la raíz del deseo?"1

Se pueden distinguir claramente en la exposición diversas preocupaciones teóricas, además de la epistemológica. Nuestra reflexión se centra sólo en un aspecto del estatuto del psicoanálisis, a saber, en la caracterización que Ricoeur hace de la especificidad del mismo y su distinción radical de la fenomenología. De manera general, podemos sintetizar esta tesis diciendo que la irreductibilidad del discurso psicoanalítico, aquello por lo que no puede ser considerado una fenomenología, radica en su propia praxis. La praxis psicoanalítica fue designada por Freud como "técnica". Para Ricoeur la técnica lleva consigo una serie de rasgos exclusivos que no pueden ser abordados de acuerdo con el método fenomenológico: "en cuanto praxis irreductible a cualquier otra- el psicoanálisis «señala con el dedo» lo que la fenomenología jamás llega a captar en forma exacta". ${ }^{2}$ Así entonces, observamos que la razón de su distinción epistemológica consiste en una cuestión metodológica: la praxis de la fenomenología es diferente de la praxis de la técnica psicoanalítica.

Nuestra reflexión parte de esta tesis de Ricoeur, pero intenta ir más allá de ella. Intentaremos mostrar que la recepción del psicoanálisis de Freud en Donald Winnicott produjo un desplazamiento epistemológico cuando dejó de lado el concepto de técnica y lo reemplazó por la noción de tratamiento. El lugar que ocupa el tratamiento psicoanalítico en el pensamiento de Winnicott

\footnotetext{
${ }^{1}$ Ricoeur, Paul, Frend. Una interpretación de la cultura, México, Siglo XXI, 1985, p. 2.

${ }^{2}$ Ibidem, p. 366.
} 
lleva consigo algo más que un mero cambio de vocabulario. Más bien se trata de un cambio epistemológico. Desde el interior del pensamiento de Winnicott se torna necesario pensar nuevamente las relaciones epistemológicas entre fenomenología y psicoanálisis.

La exposición tendrá el siguiente recorrido: primero, haremos una reconstrucción de la argumentación de Ricoeur en torno al concepto de técnica psicoanalítica. A continuación, expondremos el lugar que ocupa el tratamiento en Winnicott, tomando en cuenta las diferencias que establece con Freud. Por último, y a modo de conclusión, sacaremos las consecuencias epistemológicas que están implicadas en el pensamiento del psicoanalista inglés.

\section{Desarrollo}

La tesis epistemológica central de Ricoeur radica en que el psicoanálisis es un discurso mixto que articula una hermenéutica y una energética. La hermenéutica da cuenta de un aspecto del objeto de estudio de Freud. Los sueños, los actos fallidos, los chistes y los síntomas neuróticos pertenecen al plano del sentido y, como tales, pueden ser abordados. Es decir, pueden ser interpretados en el medio de la articulación significativa de la experiencia. Para decirlo negativamente: no son mudos. Por el contrario, se muestran en el medio de actos que dan sentido. La energética, por su parte, expresa otro punto de vista contrario al anterior. El objeto del psicoanálisis no puede ser reducido totalmente a la articulación significativa. Hay algo que está más allá del sentido y que se resiste a ingresar a sus dominios. La teoría de las pulsiones se mueve en plano de una energía que se incardina no en el plano de una intencionalidad concebida como el medio universal de la donación (Husserl), sino en una realidad que es muda, ciega y que, no obstante, irrumpe en el dominio del sentido para desarticularlo.

El psicoanálisis necesariamente tiene esta estructura paradójica. Su especificidad no consiste en la afirmación de alguno de sus polos, sino más bien en la tensión de ambos. Lo distintivo, aquello que hace que el psicoanálisis no sea ni psicología, ni medicina, ni filosofía, sino justamente psicoanálisis, es esta peculiar síntesis de su objeto que pone en juego una dimensión visible y otra invisible. Ahora bien, cuando Ricoeur analiza detalladamente la teoría de las pulsiones, matiza esta primera aproximación. Un dominio energético puro, 
una mera fuerza natural haría del concepto de pulsión una cosa que pertenecería al campo de las ciencias naturales. Una energía de esta índole tendría la capacidad de ser cuantificada, mensurada, tratada de acuerdo con los métodos cuantitativos de tales disciplinas. Pero, si bien Freud habla de energía, de desplazamiento de la misma, nunca la aborda de esta manera. Se trata de una cantidad sin número. ${ }^{3}$

Es a raíz de esta peculiaridad de la dimensión cósica del psicoanálisis que Ricoeur propone una interpretación del concepto de Repräsentanz (presentación de la pulsión) muy interesante. El psicoanálisis no se enfrenta con la pulsión desnuda, con fuerzas naturales que carecen de mediación significativa, sino más bien con los representantes psíquicos de estas fuerzas. Así lo dice Ricoeur literalmente: "La realidad que la tópica puede conocer es la de las presentaciones psíquicas de la pulsión, y no la de las pulsiones en sí mismas. Un realismo empírico no es un realismo de lo incognoscible, sino de lo cognoscible. Pues bien, lo cognoscible en psicoanálisis no es el ser biológico de la pulsión, sino el ser psicológico de las presentaciones psíquicas de la pulsión". 4

El representante psíquico ya no es una cosa, sino tiene un estatuto semiótico determinado, a saber, hace las veces de traductor-filtro entre lo natural y el sentido. Como discurso fronterizo trabaja siempre con conceptos que tienen la estructura del traductor bilingüe: hablan al mismo tiempo el lenguaje de la fuerza y el lenguaje del sentido. La mixtura constitutiva del discurso psicoanalítico no debe concebirse como si fuera una amalgama de ciencia natural y ciencia humana. Más bien lo que sucede es que claramente se posiciona dentro de las ciencias humanas, razón por la cual, tiene el estatuto de una hermenéutica. Pero lo específico dentro de este campo epistémico es que desde el mismo plano del sentido intenta dar cuenta del carácter de cosa del psiquismo humano. La energética como condición constitutiva del psicoanálisis no es algo ajeno a la hermenéutica, sino que desde el mismo plano del sentido da cuenta del sinsentido, de aquello que no puede reducirse a la significación, de lo natural de la subjetividad humana. Refiriéndose a la interpretación lacaniana de Freud, Ricoeur afirma:

\footnotetext{
3 Ibidem, p. 67.
}

${ }^{4}$ Ibidem, p. 380. 
Por eso nos parecía que una interpretación puramente lingüística del análisis elude la dificultad fundamental propuesta por Freud; su naturalismo está "bien fundamentado"; y eso que fundamenta es el aspecto de cosa, de cuasinaturaleza que ofrecen las fuerzas y los mecanismos estudiados. Si no llegamos hasta ahí, tarde o temprano volvemos a parar en la primacía de la conciencia inmediata. ${ }^{5}$

Esta tesis general sobre el estatuto mixto del psicoanálisis es el marco donde Ricoeur aborda el concepto de técnica. El problema que orienta la indagación es nuevamente la cuestión de la especificidad del psicoanálisis frente a la fenomenología. En efecto, si el psicoanálisis se enfrenta con fenómenos, objetos articulados significativamente, entonces se mueve dentro del campo del análisis intencional de la fenomenología. Ricoeur acepta que hay varios aspectos comunes con la fenomenología, pero se esfuerza también por dar razones para decir que hay algo irreductible a ella. Nos interesa referirnos ahora a las razones por las que el psicoanálisis está más allá de la fenomenología o, para decirlo en los propios términos de Ricoeur, por las que se presenta como una antifenomenología. ${ }^{6}$ Ciertamente que no abordaremos todos los aspectos de este problema, sino tan sólo aquellos que hacen del psicoanálisis un juego del lenguaje sui generis.

Ricoeur compara al psicoanálisis y la fenomenología desde cuatro puntos de vista. La reducción fenomenológica, en primer lugar, muestra que la vía de acceso al campo de investigación de la conciencia se lleva a cabo como un acto de libertad. En cambio, el psicoanálisis tiene en el punto de partida algo así como una reducción invertida. ${ }^{7}$ En efecto, el acto inaugural del psicoanálisis, por decirlo así, consiste en suspender la conciencia como libertad para hacer comparecer las motivaciones inconscientes más profundas y de este modo ver que la conciencia no más que un esclavo del inconsciente. ${ }^{8}$ En segundo lugar, la fenomenología no puede dar cuenta, desde del punto de vista metodológico,

\footnotetext{
${ }^{5}$ Ibidem.

${ }^{6}$ Ibidem, p. 370.

${ }^{7}$ Ibidem, p. 108.

${ }^{8}$ Ibidem, p. 241.
} 
del inconsciente freudiano ya que no conoce una instancia separada de la conciencia por la barrera de la represión y, menos aún, puede analizar la distorsión que sufren las formaciones del inconsciente cuando cruzan la frontera de la represión: "Por eso hace falta otra técnica para entender aquel alejamiento y aquella disociación en que se basan la distorsión y la sustitución que hacen irreconocible el texto de la conciencia". ${ }^{9}$ Lo reprimido como el rasgo constitutivo del inconsciente requiere de una técnica sui generis que está más allá del método fenomenológico. En tercer lugar, Ricoeur se refiere a la interpretación lacaniana del inconsciente estructurado como un lenguaje. Tampoco esta lectura hace que el psicoanálisis pueda ser considerado totalmente como una fenomenología. Ricoeur toma como punto de partida la lingüística de la enunciación de Benveniste para tomar posición frente a la tesis de Lacan. El inconsciente freudiano más que un lenguaje en sentido estricto tiene que ser caracterizado como un cuasi lenguaje. En efecto, el psicoanálisis no se enfrenta con "fenómenos organizados en la lengua", 10 sino con estructuras infra y supralingüísticas. El simbolismo del sueño es infralingüístico por dos razones: a) opera al nivel del desplazamiento y la condensación de imágenes y no de articulaciones significativas, y b) es un simbolismo que atraviesa diversas culturas independientemente de la lengua que se hable en dicha cultura. Por otro lado, el análisis del simbolismo del sueño muestra que se trata de fenómenos supralingüísticos en la medida en que se expresa en unidades del discurso superiores al enunciado que, según Benveniste es el límite superior del lenguaje, tales como el proverbio, dichos, mitos, folklore. ${ }^{11}$

Más precisamente, el inconsciente freudiano se presenta como un mecanismo que permanentemente confunde lo infralingüístico con lo supralingüístico. ${ }^{12}$ Justamente porque el psicoanálisis se enfrenta con fenómenos cuasi-lingüísticos no puede ser considerado como una fenomenología. El método fenomenológico no posee los instrumentos adecuados para dar cuenta de esta distorsión constitutiva del inconsciente. ${ }^{13}$ Por último, Ricoeur aborda el problema de la intersubjetividad. A su juicio, es

\footnotetext{
${ }^{9}$ Ibidem, p. 344.

${ }_{10}$ Ibidem, p. 348.

${ }^{11}$ Ibidem, pp. 348-349.

12 Ibidem, p. 349.

${ }^{13}$ Ibidem, pp. 354-355.
} 
en este plano dónde se ve con mayor claridad por qué el psicoanálisis no es una fenomenología. La razón de ello está en que Freud considera que el vínculo intersubjetivo entre analista y analizante es una relación técnica. Para Ricoeur esta afirmación es el gesto más audaz de Freud:

es aquí [se refiere a la intersubjetividad] donde el psicoanálisis se distingue en forma más radical de todo cuanto la fenomenología puede comprender y producir con los solos recursos de la reflexión. Diferencia que yo resumo en una sola palabra: el psicoanálisis es una técnica ardua, que se aprende con el ejercicio y la práctica asidua. Jamás nos sorprenderemos bastante de tan audaz hallazgo: tratar la relación intersubjetiva como una técnica. ${ }^{14}$

Es una técnica en el sentido de que es un trabajo llevado a cabo por analista y analizante en donde se descubre el mecanismo de la neurosis. Concebida como un trabajo, la técnica psicoanalítica se organiza en torno a tres conceptos que están completamente ausentes en la fenomenología: resistencia, transferencia y repetición. ${ }^{15}$

En un artículo publicado en el 1964, un año antes que el libro sobre Freud, Ricoeur expone de una manera mucho más detallada el concepto de técnica psicoanalítica desde el punto de vista del vínculo intersubjetivo entre analista y analizante. El artículo se titula "Técnica y no técnica en la interpretación". Lo interesante de este trabajo radica no sólo en que en la noción de trabajo adquiere una mayor precisión, sino fundamentalmente en que Ricoeur sostiene que la técnica psicoanalítica cae por fuera de la noción moderna de tecnología como dominación de la naturaleza. El concepto de trabajo como hilo conductor del esclarecimiento de la noción de técnica tiene como finalidad sostener que el psicoanálisis es una forma de la praxis a la que Ricoeur le asigna el término griego de téchne ${ }^{16}$ y que dicha praxis se diversifica, por un lado, en la práctica de la interpretación (hermenéutica) y, por otro, en la

\footnotetext{
${ }^{14}$ Ibidem, p. 355.

15 Ibidem, p. 356-363.

16 "Técnica y no técnica en la interpretación” en Ricoeur, P., El conflicto de las interpretaciones. Ensayos de hermenéutica, Buenos Aires, FCE, 2003, p. 167.
} 
elaboración teórica de los mecanismos psíquicos que fundamentan la interpretación (metapsicología). Desde la perspectiva hermenéutica de la técnica psicoanalítica el trabajo aparece como una labor que el analista hace cuando lucha contra las resistencias del analizante y al que le corresponde una elaboración (durcharbeiten) de dichas resistencias por parte del paciente. ${ }^{17}$ En este doble trabajo contra las resistencias se ponen en juego las fuerzas de la transferencia. Por eso dice Ricoeur: "la disciplina del análisis es, en lo esencial, una disciplina de la satisfacción, todo el manejo consiste en utilizar el amor de transferencia sin satisfacerlo." 18 Desde la perspectiva metapsicológica el concepto de trabajo aparece en la descripción del psiquismo. Trabajo de sueño, trabajo de desplazamiento, trabajo de condensación dan cuenta de que el psiquismo es un artefacto, una máquina cuyo mecanismo fundamental es la distorsión del deseo. El psiquismo se presenta, así, como una técnica del disfraz. ${ }^{19}$

Ahora bien, la praxis del psicoanálisis en sus dos vertientes no puede ser equiparada al modo en que la ilustración concibió la técnica. No se trata de una praxis humana cuya finalidad primordial sea el dominio de la naturaleza humana. Justamente para indicar que está por fuera de la lógica de la dominación, Ricoeur crea una expresión paradójica: la técnica psicoanalítica es una antitécnica. ${ }^{20} \mathrm{La}$ razón de ello está en que el psicoanálisis no trata con un objeto natural, sino con la verdad del sujeto. Su modelo es Edipo Rey. El ámbito donde se despliega la técnica de la veracidad es la palabra. El trabajo del psicoanálisis no más que trabajo del lenguaje. ${ }^{21}$ Pero fundamentalmente el psicoanálisis no consiste en una técnica de dominación porque tiene una intención liberadora muy cercana a la ética de Spinoza. El psicoanálisis nos devuelve la libertad no como libre albedrío, sino como liberación:

${ }^{17}$ Ibidem, pp. 166-168.

${ }^{18} \mathrm{Ibidem}$, p. 169. Más adelante, Ricoeur comenta que las fuerzas de la transferencia es lo que hace del psicoanálisis un discurso irreductible a la reflexión de la fenomenología (Ibidem, p. 168).

${ }^{19}$ Ibidem, p. 171.

${ }^{20}$ Ibidem, p. 172.

${ }^{21}$ Ricoeur se remite a Lacan (Ibidem, p. 174). 
"Gustosamente situaré esta liberación bajo dos emblemas: poder hablar, poder amar". 22

\section{Técnica y tratamiento en el psicoanálisis de $\mathrm{D}$. W. Winnicott}

Desde los señalamientos de Ricoeur, el psicoanálisis aparece como una praxis específica no reductible a la fenomenología. Es a partir de su técnica que la clínica puede orientarse hacia una experiencia de libertad. Freud siempre insistió a lo largo de su obra que la eficacia del caso se sostiene en la obediencia del analista a la técnica. Su buen manejo es que la garantiza no sólo la validez del discurso psicoanalítico, sino también su ética. Esta premisa se mantuvo en autores posteriores a Freud como M. Klein y J. Lacan. Sin embargo, otros analistas proponen un vocabulario diverso para describir la clínica. Entre ellos, se destacan los aportes de D. W. Winnicott. Para Winnicott, el analista debe acompañar al sujeto a la emergencia de los fenómenos transicionales, entendidos como la manifestación del sí mismo, de la actividad creadora y de la expresión del propio ser. En función de ello, la praxis se orienta, ya no como la especificidad de una técnica, sino como un espacio de juego entre dos. Esta diferenciación tiene consecuencias epistemológicas, al punto tal que técnica y tratamiento ya no son similares. Para justificar esta afirmación haremos el siguiente recorrido, de acuerdo con los ejes presentados por Ricoeur en su lectura de Freud. Estos son la posición del analista y la diferencia entre técnica y tratamiento.

\subsection{La posición del analista}

A lo largo de los escritos técnicos como "Sobre la dinámica de la transferencia" (1912), "Consejos al médico" (1912), "Trabajo sobre la técnica psicoanalítica" (1914), "Conferencias de introducción al psicoanálisis" (1916), entre otros, Freud se esfuerza por establecer las coordenadas de la praxis a partir de la instrumentalización de la regla fundamental y de la posición del

22 Ibidem, p. 178. 
analista, cuya función es idéntica a la del cirujano. ${ }^{23}$ Tal como lo establece Assoun, "Freud hace de su método de investigación y de su clínica, al analista un científico de los procesos psíquicos". ${ }^{24}$ Winnicott, por el contrario, establece que en la relación analista-paciente, se debe sostener en la confianza de un ambiente sostenedor, vital, semejante a los primeros cuidados maternos.

En este tipo de relación terapéutica, la instalación de la transferencia habilita a que sea la noción de cuidado, entendido como sinónimo de cura (care) la que establezca el modo de desarrollo del proceso analítico. A diferencia de la imagen freudiana, el analista winnicottiano interviene activamente como sostén a través de dos términos que son holding y handling. Estas no apuntan exclusivamente para la interpretación de lo inconsciente, sino que su propósito es construir un marco de confiabilidad para que la interpretación se lleve a cabo. ${ }^{25}$ Este viraje obedece a que lo inconsciente no tiene la misma relevancia que en el psicoanálisis freudiano. El lugar más destacado y basal es el de la existencia auténtica o propio ser (true self), cuya relevancia se extiende desde las neurosis de transferencia hacia las psicosis y los trastornos borderlines. El acento no está en la determinación inconsciente sino en la posibilidad de experimentarse siendo. Las motivaciones reprimidas adquieren significación en la medida que la experiencia de sentirse "vivo, verdadero y real" se convierten en el objetivo de todo tratamiento. Este corrimiento va de la sobredeterminación inconsciente en Freud hacia al sentido de la propia existencia en Winnicott. Según Coloma Andrews "el psicoanálisis de Winnicott se orienta a tratar esa

\footnotetext{
23 "No sé cómo encarecería bastante a mis colegas que en el tratamiento psicoanalítico tomen por modelo al cirujano que deja de lado todos sus afectos y aún su compasión humana y concentra sus fuerzas espirituales en una meta única; realizar una operación lo más acorde posible a las reglas del arte" (Freud, S. "Consejos al médico" en Obras completas, Tomo XII, Buenos Aires, Amorrortu, 1996 (1912), p. 114)

${ }^{24}$ Assoun, P-L., Figuras del psicoanálisis. Buenos Aires, Prometeo, 2006, p. 123.

25 "El análisis no consiste sólo en interpretar el inconsciente reprimido, consiste en propiciar un marco confiable donde la interpretación pueda llevarse a cabo" (Winnicott, D. W., El hogar, nuestro punto de partida, Buenos Aires, Paidós, 2006, p. 133)
} 
totalidad entre ser y actuar, entre ser y hacer, y por esto considero su acción como paradójica". ${ }^{26}$

Así como Freud aconsejaba aferrarse a la técnica para no caer en la trampa de la transferencia, Winnicott entiende que debe ser flexible de acuerdo con las condiciones del caso. El uso de la técnica debe adaptarse junto con el de la interpretación. Si la interpretación en la neurosis se vuelve el modelo de todo tratamiento, la clínica de la psicosis muestra su límite. Aquí no tiene sentido la interpretación de lo reprimido, sino la posibilidad de establecer continuidad en la experiencia del ser. Frente a estos cambios sobre el quehacer del analista, la imagen ya no será la del cirujano, sino la del músico:

La única compañía que dispongo cuando me interno en un caso es la teoría que siempre está conmigo, que se ha constituido en parte de mi ser y a la que ni siquiera necesito recurrir de modo deliberado (...) Podría comparar mi situación con la de un cellista que, sólo después de transitar el arduo sendero de la técnica, y una vez que ésta se da por supuesta, se halla en condiciones de hacer música (...) deseo comunicarles a aquellos que se encuentran este duro camino de la técnica, transmitirles las esperanzas de que algún día podrán hacer música. ${ }^{27}$

Esta metáfora del cellista señala que la condición de la clínica no se sostiene en la aplicación de una técnica, sin más recursos que la simple ejecución. La técnica en el tratamiento es condición necesaria, pero no suficiente. Algo más aparece que la transforma en música. Será la particularidad del caso la que determine el uso de determinada técnica, siendo ésta flexible a las condiciones del diagnóstico. Para Winnicott no alcanza con manipular instrumentalmente las herramientas del psicoanálisis, sino que debe estar al servicio de un desafío mayor. Si en Freud la imagen del cirujano otorgaba una

${ }^{26}$ Coloma Andrews, J., "El oficio de lo invisible (una paradoja psicoanalítica)", en Outerial, F. y Abadi, S. (comp.), Donald Winnicott en América Latina: teoría y clínica psicoanalitica, Buenos Aires, Lumen, 2000, p. 58.

${ }^{27}$ Winnicott, D. W., Clínica psicoanalítica infantil, Buenos Aires, Paidós, 1993, p. 14. 
distancia relevante entre analista y paciente; en Winnicott se orienta hacia una posición vital del que se espera una elevación de la técnica. Esto produce dos cambios importantes: por un lado, que la técnica pierde paridad frente al tratamiento; y, por el otro, que se genera una apertura clínica desde el campo de las neurosis de transferencia hacia la psicosis y trastornos borderlines.

\section{2: Técnica y tratamiento}

En la obra freudiana la técnica hace al tratamiento, al punto tal que una desviación de la primera anula la consideración de un psicoanálisis en sentido estricto. Si en Freud la interpretación es el bisturí del analista-cirujano, lo es en la medida que habilita el acceso a las motivaciones inconscientes. Su función es hacer consciente lo reprimido y poner al descubierto las resistencias que aparecen disfrazadas en la transferencia. Bajo estas premisas el psicoanálisis se ubica como una "ciencia de lo inconsciente". Para reforzar esta idea cientificista, Freud adopta otra figura que es la del químico. ${ }^{28}$ Estos modelos de referencias tienen el sentido del dominio de la naturaleza mediante la técnica.

En la propuesta del psicoanalista inglés, en cambio, es el tratamiento el que define las posibilidades, ahora en plural, del tipo de técnica necesaria para abordar el caso clínico. La diferencia entre técnica y tratamiento es a partir del uso de la interpretación y las variabilidades de la clínica.

La interpretación tiene en Winnicott un interés colaborativo. ${ }^{29}$ Aquí el analista no se limita a revelar al paciente el significado oculto de lo inconsciente, sino que utiliza la interpretación para acompañar el sentido de lo personal. Martínez entiende que, para Winnicott, su primer sentido "radica en el

\footnotetext{
28 "El psicoanalista sabe que trabaja con las fuerzas más explosivas y que le hacen falta la misma cautela y escrupulosidad del químico" (Freud, S., "Puntualizaciones sobre el amor de transferencia", en Obras Completas, Vol. XII, Buenos Aires, Amorrortu, 1996, p. 173)

29 "Él (por Winnicott) entiende que sería un pecado del analista hacer interpretaciones inteligentes, aun cuando fuesen correctas, si sólo son productos de la fría aplicación de la teoría" (Anfusso, A. e Indart, V., ¿De qué hablamos cuando hablamos de Winnicott?, Montevideo, Waslala Editorial, 2009, p.221).
} 
reconocimiento del gesto comunicativo del paciente y, por lo tanto, en el reconocimiento del paciente como 'persona". 30

Aparece así una dimensión ética en la labor analítica, el cuidado por el paciente y la prudencia en la interpretación, que tiene condiciones muy distintas a la de ser una especie de traductor u oráculo de las motivaciones inconscientes. Tampoco puede verse aquí la imagen del analista como cirujano. Por el contrario, oficia como anfitrión y hacia una experiencia singular que incluye lo inconsciente, pero que no se limita únicamente a él. Es como si diera un paso más atrás y dijera "aquí hay una persona". Es decir, señala la condición misma de la existencia. Parecerá obvio en cuestiones neuróticas, pero adquiere un profundo sentido en las perturbaciones psicóticas, donde la despersonalización suele causar estragos. La interpretación, entonces, no se trata tanto de sagacidad como de compromiso. Aquí surge la idea de un horizonte primigenio, en donde Winnicott sostiene su praxis, que es el de reconocer lo que está allí. La interpretación del conflicto psíquico, por poner un ejemplo, se manifiesta sobre este escenario primordial de la existencia.

Sin embargo, la interpretación da un paso más y muestra la eventualidad de que el analista "falle". De esta manera: el estado de dependencia que conlleva la transferencia es condición para que el paciente pueda atravesar un análisis, pero no quedar anclado a él. Todo análisis tiene que tener un fin. Esto depende de los manejos del analista, en el sentido de que también es condición fallar, vale decir, oportunamente, para que aparezca la novedad del lado del paciente. La idea es contrarrestar cualquier quimérica seducción que dé indicios de omnipotencia. "Al interpretar, el analista muestra cuánto y cuán poco puede recibir de la comunicación del paciente". ${ }^{31}$ Las fallas del analista apuntan a que el paciente pueda innovar desde sí mismo. No en cualquier momento -de allí la importancia del timing del análisis. Del mismo modo que lo transicional aparece en el proceso de ilusión-desilusión entre la madre y el niño, el analista desilusiona sobre la promesa de saber: "Interpreto, porque si no lo hago, el paciente tiene la impresión de que entiendo todo". 32

\footnotetext{
${ }^{30}$ Martínez, H., Donald Winnicott en el movimiento psicoanalitico, Mar del Plata, EUDEM, 2007, p. 128.

31 Winnicott, D. W., Realidady Juego, Buenos Aires, Gedisa, 2007, p. 101.

${ }^{32}$ Ibidem, p. 125.
} 
Sobre el segundo punto, la variabilidad de la clínica, es donde más se aprecia la diferencia entre técnica y tratamiento: "Es posible llevar a cabo un tratamiento con una técnica limitada y, a la inversa, con una técnica altamente evolucionada es posible fracasar en la puesta en práctica del tratamiento". 33

Si en Freud técnica y tratamiento constituían la misma cosa, para Winnicott es la técnica la que se supedita al tratamiento, y de ser necesario, se transforma según el cuadro de la praxis. Así es posible entender que el tratamiento con psicóticos y trastornos borderlines se trate de técnica modificada: "hay muchas variedades en la clínica, su existencia depende de los requerimientos del paciente o del caso". ${ }^{34}$ Esta apertura en la frontera de la neurosis hacia diferentes formas de clínica es lo que lo lleva a interesarse en otras maniobras de la técnica adaptables a las necesidades del paciente. Según Phillips, esta subversión del tratamiento por sobre la técnica, hace de ésta una cuestión elástica coincidente con la maleabilidad del analista. ${ }^{35}$

Para Winnicott, en definitiva, el psicoanálisis es "una larga, larguísima recepción de una historia". Es la posición enunciativa del paciente, que llega al tratamiento con su propio pasado, la que ordena el modo de acercamiento hacia éste. Lo principal se define en la posibilidad de arribar a la creatividad y a la experiencia personal. Este giro modifica la relación entre analista y paciente. La relación vital marca el modo de vínculo terapéutico en términos de confiabilidad. No se trata de negar la importancia de lo inconsciente reprimido, sino ubicar las coordenadas en las que éste se sostiene. Como se ha mencionado, su propuesta de análisis incluye todos aquellos fenómenos que remiten a la problemática incertidumbre entre ser y existir. No se trata de que rechace el factor del síntoma freudiano, la rivalidad edípica, el problema del deseo y su satisfacción. Al igual que Freud, hace hincapié en la idea ficticia de una vida normal, pero esta concepción se encuentra más ligada a problemáticas tales

\footnotetext{
33 Winnicott, D. W., Escritos de pediatría y psicoanálisis, Barcelona, Laia, 1979, p. 378.

34 Winnicott, D. W., El hogar, nuestro punto de partida, p. 117. No hay que olvidar que Winnicott clasifica la variabilidad clínica de acuerdo con las experiencias favorables o desfavorables de las etapas tempranas. De acuerdo a ellas se distinguen es pacientes neuróticos, privados (psicosis) y deprivados (borderlines)

35 Phillips, A., Winnicott, Buenos Aires, Lugar Editorial, 1997, p. 57.
} 
como la inautenticidad y el sentimiento fútil de la existencia. Winnicott pone el acento en donde estos factores se sostienen: la problemática de la facticidad del siendo. En todo caso, la clínica freudiana podría leerse como un derivado del existenciario winnicottiano. Para Winnicott lo radical es la continuidad de la existencia, a partir de donde un sujeto comienza a ser. La diferencia que Winnicott menciona es que, en los primerísimos estadios, el problema no está frente al deseo, sino ante la necesidad de existir. Aquí, resulta indispensable la existencia de otro que cobije y sostenga, aunque no se tenga conciencia alguna de ello. Es esa la experiencia que retorna en el encuadre analítico. La performance del cellista muestra que lo importante no es dominar la técnica sino que a partir de ella, ejecutar música. Esta diferencia es la que se que establece entre técnica y tratamiento, lo que, en definitiva, se orienta hacia una experiencia de libertad para el paciente, en la medida que pueda arribar a la creatividad de los fenómenos transicionales

\section{Conclusiones}

En este trabajo se ha hecho un doble recorrido. Por un lado, se ha prestado atención al análisis que P. Ricoeur hace del psicoanálisis de S. Freud. Según el filósofo francés, la irreductibilidad del discurso psicoanalítico, aquello por lo que no puede ser considerado una fenomenología, radica en su propia praxis. La praxis psicoanalítica fue designada por Freud como "técnica". Para Ricoeur, la técnica lleva consigo una serie de rasgos exclusivos que no pueden ser abordados de acuerdo con el método fenomenológico, a saber, resistencia, transferencia y repetición. La razón de su distinción epistemológica consiste en una cuestión metodológica: la praxis de la fenomenología es diferente de la praxis de la técnica psicoanalítica. Resulta interesante que Ricoeur, al afirmar que el dominio que el psicoanálisis hace de la naturaleza no se refiere a la de la ciencia, no haya tenido en cuenta la figura del cirujano que propone Freud. Justamente, la comparación que realiza Freud, sumado a su pretensión de ubicar al psicoanálisis como una Natumvissenschaft muestra todo lo contrario: el análisis y el analista se realizan bajo el predominio de una disciplina que intenta captar la naturaleza del inconsciente al cual examina e interpreta. Por el contrario, el modelo que propone Winnicott del analista como chelista o como madre suficientemente buena, acerca al psicoanálisis al discurso fenomenológico del 
cuidado y la libertad. El análisis winnicottiano se orienta hacia una interpretación de la cura (care) como un entorno facilitador que cuida del padecer psíquico. Dicho cuidado tiene como horizonte la posibilidad del vivir creador, término que sólo puede realizarse sobre la base de la libertad. En efecto, si el fin de análisis en Winnicott se dirige hacia la transicionalidad, cuya base es lo creativo y espontáneo del propio ser, provoca, además de un cambio de vocabulario respecto del freudiano, un cambio epistémico. Los términos de true-self, creatividad, holding, handling, cuidado indican un nuevo sustrato metapsicológico ya no basado en el modelo de la ciencia, modelo en el cual se reflejó Freud. Por el contrario, comparte en su base términos de orientación filosófica.

Un ejemplo de ello, es el carácter universal de la transicionalidad que aparece de un modo peculiar en el campo de la patología. En efecto, cuando las circunstancias no son propicias para su expresión, el espacio potencial se modifica. De allí que la clínica en la que Winnicott trabaja no aborde tanto las cuestiones del inconsciente, sino la problemática del ser y los diversos modos en que el sí mismo habita el mundo junto con otros. Esta posición clínica dentro del campo del psicoanálisis tiene una consecuencia a nuestro entender aún mayor. Al proponer un punto de análisis a partir de la creatividad y la transicionalidad da un paso más allá de la tópica freudiana. En lugar de ello, insinúa un tercer espacio que no responde al carácter genético pulsional, sino a una relación entre el hombre y el mundo basado en la correlación mutua. Esta correlación tiene su origen en la posibilidad o no de experimentarse siendo, lo que sugiere una nueva teoría de los lugares psíquicos. Así entendido, invita a pensar una tercera tópica, sostenida en este tercer espacio potencial donde Winnicott ubica a la vida y al lugar "donde pasamos la mayor parte del tiempo".

A partir de aquí se establece un nuevo tipo de diálogo entre el psicoanálisis y la fenomenología hermenéutica.

\section{Bibliografía}

Anfusso, A. e Indart, V., ¿De qué hablamos cuando hablamos de Winnicott? Montevideo, Waslala editorial, 2009.

Assoun, P-L., Figuras del psicoanálisis. Buenos Aires, Prometeo, 2006. 
Coloma Andrews, J., "El oficio de lo invisible (una paradoja psicoanalítica)", en Outerial, F. y Abadi, S. (comp.), Donald Winnicott en América Latina: teoría y clínica psicoanalítica, Buenos Aires, Lumen, 2000.

Freud, S., "Puntualizaciones sobre el amor de transferencia" (1914) en Obras completas, Tomo XII, Buenos Aires, Amorrortu, 1996.

Freud, S., "Consejos al médico" (1912) en Obras completas, Tomo XII, Buenos Aires, Amorrortu, 1996.

Martínez, H., Donald Winnicott en el movimiento psicoanalitico, Mar del Plata, EUDEM, 2007.

Phillips, A., Winnicott, Buenos Aires, Lugar Editorial, 1997.

Ricoeur, P., Frend. Una interpretación de la cultura, México, Siglo XXI, 1985.

Ricoeur, P., "Técnica y no técnica en la interpretación" en El conflicto de las interpretaciones. Ensayos de hermenéutica, Buenos Aires, FCE. 2003.

Ricoeur, P., "Image et Langage en Psychanalyse" en Écrits et Conférences, tomo 1 : Autour de la psychanalyse, Paris, Éditions du Seuil, 2008.

Winnicott, D. W., Escritos de pediatría y psicoanálisis, Barcelona, Laia, 1979.

Winnicott, D. W., Clínica psicoanalítica infantil, Buenos Aires, Paidós, 1993.

Winnicott, D. W., El hogar, nuestro punto de partida, Buenos Aires, Paidós, 2006.

Winnicott, D. W., Realidady Juego, Buenos Aires, Gedisa, 2007.

Recibido: 05/2018; aceptado: 10/2018 